\title{
Monitoramento da floresta a curto prazo em área de concessão florestal na Amazônia ocidental
}

A concessão florestal foi implementada com o objetivo de promover a conservação dos recursos naturais e ao mesmo tempo gerar o desenvolvimento socioeconômico. O monitoramento da floresta após exploração é uma ferramenta importante para a tomada de decisões no manejo florestal. Diante disso, o objetivo deste estudo foi avaliar os efeitos da exploração na estrutura da floresta após exploração sob diferentes taxas de corte. 0 estudo foi realizado em uma unidade de manejo florestal, Flona do Jamari, RO, a primeira floresta em regime de concessão no Brasil. Foram monitoradas duas unidades de produção anual (UPA), com taxas de corte de $14,4 \mathrm{~m}^{3}$ ha-1 e $11,64 \mathrm{~m}^{3}$ ha-1. Amostrou-se todos os indivíduos arbóreos com $\mathrm{DAP}=10 \mathrm{~cm}$ em parcelas permanentes de $0,5 \mathrm{ha}$ aleatorizadas. As avaliações foram realizadas antes e após um ano do término das atividades de exploração. Realizou-se a análise da composição florística, estrutura fitossociológica, taxas de mortalidade, ingresso e crescimento. O Teste t foi usado para verificar diferenças estatísticas em cada ambiente. As atividades de exploração afetaram de forma significativa a composição e e densidade de plantas na UPA 6. As espécies raras e os indivíduos do sub-bosque foram os mais afetados pela exploração madeireira. $O$ aumento nas taxas de mortalidade e colheita da madeira favoreceu para que houvesse maior perda de estoque em área basal do que ganho após um ano do término da exploração florestal. O monitoramento da floresta a médio e longo prazo será importante para entender os processos dinâmicos e a recuperação das áreas afetadas pela extração madeireira.

Palavras-chave: Unidades de Conservação; Manejo Florestal Sustentável; Taxas de Corte.

\section{Short-term forest monitoring in a forest concession area in the western Amazon}

\begin{abstract}
The forest concession was implemented with the objective of promoting the conservation of natural resources and at the same time generating the socio-economic development. In this sense, monitoring the forest after logging is an important tool for decision-making in forest management. Thus, the aim of this study was to compare the effects of logging on forest structure after logging at different logging rates. The study was conducted in a forest management unit in Flona do Jamari, RO, the first forest under concession in Brazil. Two Annual production units (UPA) were monitored, with cut rates of $14.4 \mathrm{~m}^{3}$.ha- ${ }^{1}$ and $11.64 \mathrm{~m}^{3}$.ha- ${ }^{1}$. All tree individuals with $\mathrm{DBH}=10 \mathrm{~cm}$ were sampled in 0.5 ha permanent plots randomized before and after one year from the end of logging activities. Floristic composition, phytosociological structure, mortality rates, admission and growth were analyzed. The t-test was applied to verify statistical differences between the variables in each environment. The exploration activities significantly affect the composition and density of plants in the UPA 6 . The rare species and understory individuals were the most affected by logging. The increase in mortality rates and harvesting of wood favored a greater loss of stock in the basal area than gained after one year after the end of forestry. Monitoring the forest in the medium and long term will be important to understand the dynamic processes and the recovery of the areas affected by logging.
\end{abstract}

Keywords: Conservation Units; Sustainable Forest Management; Cut Rate.

Topic: Ciências Florestais

Reviewed anonymously in the process of blind peer
Received: 01/08/2020

Approved: $18 / 09 / 2020$
Suelen Tainã Silva Fagundes (DD

Universidade Federal de Rondônia, Brasil http://lattes.cnpq.br/5442926714701002 http://orcid.org/0000-0002-2187-2567

taynanfag@hotmail.com

Marta Silvana Volpato Sccoti (it)

Universidade Federal de Rondônia, Brasil http://lattes.cnpq.br/8802033326043600 http://orcid.org/0000-0001-5979-3218 martasccoti@unir.br

Ariane Cristina Rebelo Lima (it)

Universidade Federal de Rondônia, Brasil http://lattes.cnpq.br/1054553037312649

http://orcid.org/0000-0001-8839-0987

ariane.crlima94@hotmail.com

\author{
João Fideles Brito Junior (iD \\ Universidade Federal de Rondônia, Brasil \\ http://lattes.cnpq.br/4160488242507317 \\ http://orcid.org/0000-0002-5925-0667 \\ britojf.ro@gmail.com \\ Scheila Cristina Biazatti (iD \\ Universidade Federal de Rondônia, Brasil \\ http://lattes.cnpq.br/6379875277285936 \\ http://orcid.org/0000-0001-5017-9780 \\ scheilacristinabiazatti@gmail.com
}

DOI: 10.6008/CBPC2179-6858.2020.005.0006

Referencing this:

FAGUNDE, S. T. S.; SCCOTI, M. S. V.; LIMA, A. C. R.; BRITO JUNIOR, J. F.; BIAZATTI, S. C.. Monitoramento da floresta a curto prazo em área de concessão florestal na Amazônia ocidental. Revista Ibero Americana de Ciências Ambientais, v.11, n.5, p.55-62, 2020. DOI: http://doi.org/10.6008/CBPC2179-6858.2020.005.0006 


\section{INTRODUÇÃO}

As Florestas Nacionais (Flonas) são unidades de conservação de uso sustentável instituídas pela Lei Federal 9.985/2000, nas quais é possível promover o manejo florestal dos seus recursos, principalmente a madeira. Para regulamentar o uso desses ecossistemas o governo sancionou a Lei 11.284/2006 que foi o marco legal para promover a produção nessas áreas por meio da concessão florestal, onde o estado, por meio de licitação, pode conceder à empresas e comunidades o direito de manejar unidades de conservação de uso sustentável, explorando seus recursos madeireiros e não madeireiros.

A primeira Flona submetida a esse regime de uso foi a Floresta Nacional do Jamari, no estado de Rondônia, em 2010. Entre os anos de 2010 e 2018 na área já foram transportados 253.154,64 m³ de madeira de espécies nativas (SFB, 2019). A exploração florestal nessas áreas é feita por meio de técnicas de manejo florestal sustentável, com controle da taxa de corte, ciclo de corte, entre outros, sendo uma prática relativamente comum em algumas florestas tropicais da África, Ásia e América Latina (D’OLIVEIRA et al., 2017).

Assim, para avaliar o impacto gerado pelo manejo florestal e a recuperação da floresta, a legislação prevê o monitoramento da vegetação remanescente após exploração, como forma de avaliar a evolução da floresta diante das perturbações sofridas, incluindo-se os efeitos do regime de manejo e a resposta aos tratamentos silviculturais quando esses forem aplicados (BRASIL, 2009).

No entanto, alguns estudos questionam a sustentabilidade do manejo florestal na manutenção da qualidade produtiva da floresta e manutenção da biodiversidade, sendo importante utilizar técnicas silviculturais após exploração (HIRAl et al., 2012). Por outro lado, o uso de técnicas de exploração de impacto reduzido tem indicado redução de danos ao solo e a floresta remanescente e recuperação da floresta a longo prazo (DIONIOZIO et al., 2018; OLIVEIRA et al., 2019).

Porém, ressalta-se que o bioma amazônico apresenta inúmeras particularidades e conhecer as respostas a cada ambiente se torna importante para traçar um modelo de produção que respeite a manutenção da qualidade desses ambientes manejados, principalmente em áreas de unidades de conservação, que resguardam parte da biodiversidade brasileira.

Diante disso, este trabalho teve por objetivo avaliar os efeitos das diferentes taxas de corte utilizada no plano de manejo florestal em área de concessão na Flona do Jamari, RO, buscando descrever as principais alterações na composição da estrutura das espécies florestais após exploração.

\section{MATERIAIS E MÉTODOS}

\section{Localização e caracterização da área de estudo}

O estudo foi realizado em duas Unidades de Produção Anual, UPAs 5 e 6, localizadas na Unidade de Manejo Florestal (UMF) III, Floresta Nacional (Flona) do Jamari, RO. As UPAs apresentaram área de efetiva exploração de 1.596,70 ha e 1572,44 ha, com taxas de corte de 14,64 $\mathrm{m}^{3} \mathrm{ha}^{-1}$ e 11,64 $\mathrm{m}^{3}$.ha $\mathrm{h}^{-1}$, respectivamente (Figura 1). A execução do plano de manejo seguiu técnicas de exploração de impacto 
reduzido, como queda direcionada de árvores, planejamento de arraste e largura máxima da estrada de 4 metros.

O clima da região, segundo a classificação de Köppen Geiger é do tipo Am, apresentando período de seca bem definido (ALVARES et al., 2013). A temperatura média anual na região varia entre 24 e 26 @C, já a média anual de precipitação varia entre 1440 a 2600 mm.ano-1 (RONDÔNIA, 2010). O relevo da área é formado na sua maioria $(87,7 \%)$ por altitude inferior a 150 metros (AMATA, 2009). A classe de solo de maior frequência na área é Podzólicos Vermelho-Amarelo Distróficos (BRASII, 2005) e a vegetação predominante, é a Floresta Ombrófila Aberta Submontana, caracterizada como um tipo de vegetação de transição entre a Floresta Amazônica e as áreas extras amazônicas (VELOSO et al., 1991).

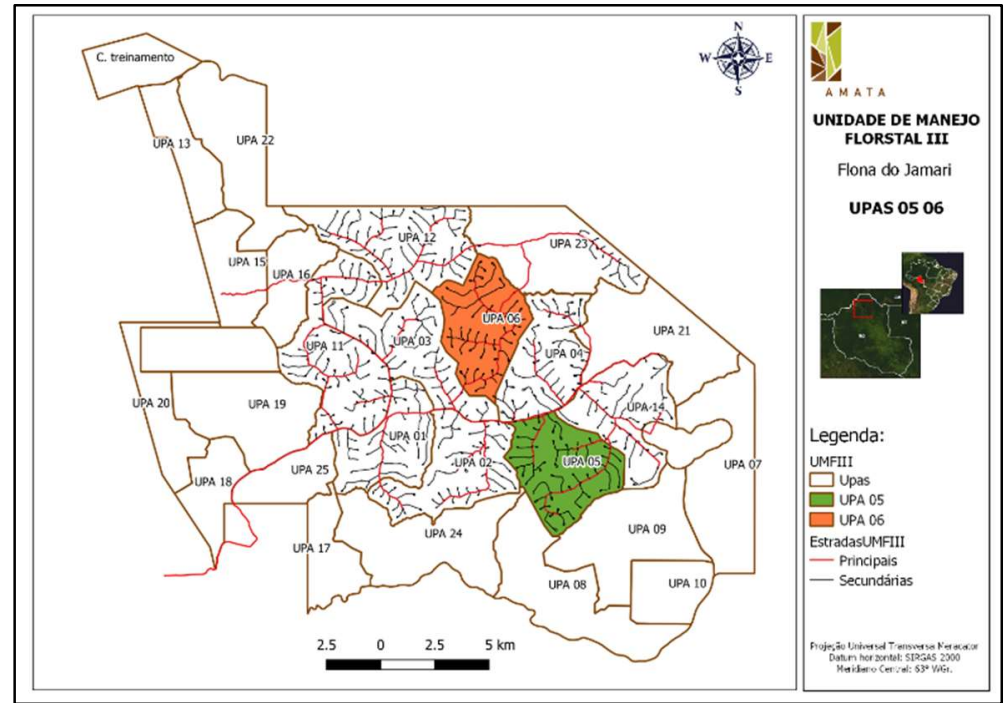

Figura 1: Localização das Unidades de Produção Anual (UPA) 5 e 6 na Unidade de Manejo Florestal III, Flona do Jamari, Rondônia.

\section{Levantamento dos dados}

Em cada UPA foram demarcadas parcelas permanentes de 50 × 100m distribuídas de forma aleatória, onde amostrou-se todos os indivíduos arbóreos com DAP $\geq 10 \mathrm{~cm}$. As parcelas foram marcadas e avaliadas, seguindo as orientações técnicas previstas nas diretrizes para instalação e medição de parcelas permanentes em florestas naturais do Bioma Amazônia (SILVA et al., 2005). Assim, foi realizado um inventário préexploratório, o qual teve por objetivo caracterizar a composição e estrutura da vegetação antes da exploração; e, o inventário pós-exploratório, utilizado para avaliar as alterações na vegetação após 12 meses do término das atividades de exploração.

No inventário pré-exploratório foram coletados dados de DAP e altura total. As espécies não identificadas tiveram seu material botânico coletado para confecção de exsicatas, e posteriormente, identificação. No pós-exploratório, remediu-se as árvores da primeira avaliação e incluiu-se os indivíduos ingressantes, bem como, o registro e causa de indivíduos mortos.

Para as espécies identificadas, fez-se a conferencia da correta nomenclatura científica, usando como referência o banco de dados da Flora do Brasil 2020 do Instituto de Pesquisas Jardim Botânico do Rio de Janeiro e também, no banco de dados do Herbário New York Botanical Garden, adotando o sistema de 
classificação Angiosperm Phylogeny Group. O material botânico coletado encontra-se no Laboratório de Ecologia e Manejo de Florestas Naturais da Universidade Federal de Rondônia-Campus de Rolim de Moura.

\section{Análise dos dados}

Para comparação das alterações ocorridas na vegetação após a exploração madeireira, realizou-se análise de riqueza florística e os índices de diversidade representados pela diversidade de Shannon e equabilidade de Pielou (SOUZA et al., 2013). Foi avaliada ainda, a estrutura horizontal por meio da densidade, dominância, índice de valor de importância e índice de espécies raras. Por último, gerou-se as taxas de mortalidade e ingresso para todas as espécies e por espécies e a análise das entradas (ingresso e crescimento) e saídas (morte e colheita) do estoque (SOUZA et al., 2013).

Os dados de composição florística, densidade, taxa de mortalidade total, ganhos e perdas de estoque, em cada UPA, foram submetidos à análise de normalidade e homogeneidade e comparados pelo Teste $\mathrm{t}$ a 95\% de probabilidade. As análises foram realizadas no programa estatístico R CORE TEAM (2019), usando o pacote Agricolae para os testes estatísticos e o Fito R para estrutura fitossociológica.

\section{RESULTADOS E DISCUSSÃO}

Com base nos inventários realizados nas parcelas permanentes das Unidades de Produção Anual (UPA) 5 e 6, notou-se redução no número de indivíduos amostrados após a exploração, sendo que na UPA 5 não houve diferença estatística $(p=0,3135)$ entre as médias geradas para o número de indivíduos por hectare antes e após exploração, já na UPA 6, observou-se diferença entre as médias ( $p$ valor=0,0132), o que também afetou o número de espécies após a exploração $(p=0,0219)$ (Tabela 1$)$. As demais variáveis avaliadas (gênero, famílias e índices de diversidade) não indicaram fragilidade diante das atividades exploratórias.

Tabela 1: Densidade, riqueza e diversidade florística observadas em parcelas permanentes nas Unidades de Produção Anual (UPA) 5 e 6, antes e após exploração na Flona do Jamari, RO.

\begin{tabular}{lcccc}
\hline \multirow{2}{*}{ Variáveis } & U.E & \multicolumn{3}{c}{ UPA 6} \\
\hline DA (ind.ha ${ }^{-1}$ ) & A.E & AP.E & A.E & $414.4^{* *}$ \\
Número de espécies* & 464.29 & 454.86 & 464 & $101^{* *}$ \\
Número de gênero & 133 & 131 & 104 & 71 \\
Número de famílias & 100 & 98 & 74 & 28 \\
H' $^{\prime}$ & 35 & 35 & 29 & 3,51 \\
J & 3,65 & 3,63 & 3,50 & 0,76 \\
\hline
\end{tabular}

Onde: * O número de espécies apresentado na tabela refere-se apenas as que foram identificadas, não foram contabilizadas morfoespécies e indivíduos não identificados. A.E: Antes da exploração, AP.E: Após exploração, DA: Densidade absoluta, $\mathrm{H}^{\prime}$ : Índice de diversidade de Shannon, J: Índice de equabilidade de Pielou, ${ }^{* *}$ Difere estatisticamente pelo teste $\mathrm{t}$ a $95 \%$ de probabilidade.

Ressalta-se que neste trabalho devido à dificuldade de coleta de material botânico, muitos indivíduos não foram identificados. Na UPA 5, 279 árvores não foram identificadas e cinco foram definidas como morfoespécies, ou seja, sabe-se que são espécies diferentes, mas não foram identificadas por nome científico. Na UPA 6146 indivíduos foram classificadas como não identificados e 84 definidas como morfoespécies. 
Apesar da UPA 6 ter apresentado menor taxa de corte $\left(11,7 \mathrm{~m}^{3} \mathrm{ha}^{-1}\right)$ verificou-se que a redução no número de indivíduos e espécies foi maior que na UPA 5, que teve taxa de corte de 14,14 $\mathrm{m}^{3} \mathrm{ha}^{-1}$. A UPA 6, teve em média a perda de 24,8 indivíduos por parcela, enquanto que na UPA 5, a média de perdas por parcela foi de 4,71 indivíduos. Ruy et al. (2014) observaram que quanto maior for a área basal explorada, maior será os danos a floresta remanescente, mesmo que seja um número menor de indivíduos danificados.

No entanto, no presente estudo, esse cenário se comportou de forma inversa, a área que sofreu maior dano foi a UPA com menor taxa de exploração. O resultado pode ser explicado pela alocação das parcelas que são distribuídas na área de forma aleatória. Percebeu-se que as parcelas da UPA 6 ficaram próximas as áreas de operação. Era comum observar ramais de arraste passando no interior das parcelas, cenário esse, que foi pouco descrito na UPA 5. Outro fato que pode ter favorecido para a maior perda de indivíduos na UPA 6 foi o abate de duas árvores (Astronium lecointei) na parcela 2. Nesta parcela observou a morte de 14 árvores em decorrência das atividades de exploração.

As espécies que foram perdidas após exploração foram aquelas com baixa densidade, denominadas como rara. Na UPA 6 observou-se maior índice de espécies raras (66\%) o que também favoreceu a maior perda de espécies nas parcelas avaliadas. Na UPA 5 o índice de espécies raras foi de 50\%.

Conforme Ivanauskas et al. (2004), espécies raras, são aquelas que ocorrem em baixa densidade. Assim, os poucos indivíduos dessas espécies se afetados pelas atividades exploratórias podem contribuir para a menor composição florística após-exploração. Na UPA 6, as espécies que foram afetadas pelas atividades de exploração estavam representadas apenas por um ou dois indivíduos, como a Byrsonima crispa, Platymiscium duckei e Tabebuia incana.

Tabela 2: Estrutura fitossociológica antes e após exploração das cinco espécies de maior valor de importância observadas nas Unidades de Produção Anual 5 e 6 com diferentes taxas de corte, na Flona do Jamari, Unidade de Manejo Florestal III, RO.

\begin{tabular}{|c|c|c|c|c|c|c|c|c|}
\hline \multirow{3}{*}{ Espécies } & \multicolumn{8}{|c|}{ UPA 5} \\
\hline & \multicolumn{4}{|c|}{ ANTES } & \multicolumn{4}{|c|}{ APÓs } \\
\hline & DA & DoA & Fabs & VI & DA & DoA & FA & VI \\
\hline Protium robustum (Swart) D.M. Porter & 49,7 & 1,9 & 100,0 & 20,7 & 48,6 & 1,9 & 100,0 & 21,0 \\
\hline Pseudolmedia spp. & 40,0 & 1,3 & 100,0 & 15,8 & 38,0 & 1,3 & 100,0 & 15,8 \\
\hline Sclerolobium spp. & 23,7 & 1,5 & 100,0 & 13,1 & 22,9 & 1,4 & 100,0 & 13,2 \\
\hline Eschweilera pseudodecolorans S.A. Mori & 24,6 & 0,9 & 100,0 & 10,6 & 24,9 & 0,9 & 100,0 & 11,3 \\
\hline \multirow[t]{2}{*}{ Pouteria torta (Mart.) Radlk. } & 26,0 & 0,7 & 100,0 & 10,4 & 24,6 & 0,7 & 100,0 & 10,2 \\
\hline & \multicolumn{8}{|c|}{ UPA 6} \\
\hline Pseudolmedia laevis (Ruiz \& Pav.) J. F. Macbr. & 53,6 & 1,9 & 100,0 & 20,0 & 46,0 & 4,6 & 100,0 & 30,2 \\
\hline Tetragastris sp. & 21,6 & 1,4 & 80,0 & 11,0 & 18,0 & 1,3 & 80,0 & 10,6 \\
\hline Peltogyne paniculata Benth. & 17,2 & 1,2 & 100,0 & 9,6 & 16,0 & 1,1 & 100,0 & 9,5 \\
\hline Tachigali chrysophylla (Poepp.) Zarucchi \& HeI & 16,4 & 1,2 & 100,0 & 9,3 & 16,4 & 1,3 & 100,0 & 10,5 \\
\hline Pouteria torta (Mart.) Radlk. & 18,0 & 0,9 & 100,0 & 8,5 & 16,4 & 0,8 & 100,0 & 8,6 \\
\hline
\end{tabular}

Onde: DA: densidade absoluta (ind.ha-1 ${ }^{-1}$, DoA: dominância absoluta $\left(\mathrm{m}^{2}\right.$.ha-1 $)$, FA: frequência absoluta (\%), VI: valor de importância (\%).

Esse cenário também foi descrito por Castro et al. (2018) ao avaliarem impactos da exploração na composição florística na Floresta Nacional do Tapajós (PA). Neste estudo, os autores constataram o desaparecimento de espécies após exploração, devido à morte de seu único representante. Quanto aos valores dos índices de diversidade de Shannon-Weaner ( $\mathrm{H}^{\prime}$ ) e equabilidade de Pielou (J) verificou-se que esses 
não foram afetados pelas atividades de exploração (Tabela 1), mantendo os valores dentro de limites normalmente encontrados em florestas tropicais manejadas (CASTRO et al., 2018; LIMA et al., 2018).

Considerando as cinco espécies de maior valor de importância, constatou-se que apenas a UPA 6 apresentou alterações na estrutura dessas espécies, onde a Peltogyne paniculata e a Pouteria torta demonstraram redução no valor de importância após exploração (Tabela 2). Isso se deu em função da atividade explorátoria, constatada nas parcelas permanentes avaliadas, ocasionando a morte de indivíduos dessas espécies, causada na sua maioria por danos provenientes da exploração (87,5\%), alterando desta forma o valor da densidade e dominância dessas espécies, e consequentemente, o valor de importância.

A taxa de mortalidade observada na UPA 5 foi de 5,61\% e na 6 de 11,78\%, sendo que as atividades de exploração foram responsáveis pelo maior quantitativo de árvores mortas nos dois ambientes (Tabela 3). Esse cenário é esperado nos planos de manejo, após os primeiros anos da colheita como constatado também por Lima et al. (2018) e Kroessin (2013).

Tabela 3: Numero de árvores mortas, ingressantes e crescimento dos indivíduos em diâmetro após a exploração nas Unidades de Produção Anual 5 e 6, antes e após exploração na Flona do Jamari, RO.

\begin{tabular}{ccccc}
\hline UPA & M.EXP & M.N & ING & IP $(\mathrm{mm})$ \\
\hline 5 & 53 & 42 & 68 & 5,3 \\
6 & 114 & 25 & 19 & 7,0 \\
\hline
\end{tabular}

Onde: M.EXP: Morta por exploração, M.N: Morte natural, ING: Ingressantes, IP: Incremento periódico em Diâmetros a altura do peito (DAP).

A maior concentração de árvores mortas ocorreu nas menores classes de diâmetro, entre 10 a 20 $\mathrm{cm}$, indicando que durante o abate os indivíduos do sub-bosque são os mais afetados. Além disso, essa classe de tamanho é que tem maior densidade de plantas, o que favorece para o elevado valor de indivíduos mortos. Esse fato também foi constatado por Bezerra et al. (2018) em um estudo na Floresta Nacional do Tapajós, onde o autor observou, que nas classes de menor diâmetro existe maior percentual de árvores mortas.

Verificou-se que as espécies com maior número de indivíduos mortos na UPA 5 foram Protium robustum e Sclerolobium spp., juntas representaram 1,06\% do total de indivíduos mortos e, na UPA 6, as espécies com maior número de indivíduos mortos foram Pseudolmedia laevis (1,70\%), Tetragastris sp. $(0,76 \%)$ e Eschweilera pseudodecolorans (0,59\%).

A taxa de ingresso, em ambos os ambientes foi menor que a de mortalidade. Na UPA 5 foram registrados um total de 68 indivíduos ingressantes, que representaram uma taxa de ingresso total de 4,18\%, com maior ocorrência das espécies Protium robustum (7,35\%) e Sclerolobium spp. (8,82\%). Enquanto que na UPA 6, foram quantificados 19 indivíduos ingressantes, apresentando taxa de ingresso de 1,63\%. As espécies com maior número de indivíduos ingressantes foram as mesmas da UPA 5, Protium robustum e Eschweilera spp.

Considerando os estoques de madeira representados pela área basal de todas as árvores amostradas, verificou-se maior perda do que entrada na floresta. No entanto sem diferenças estatísticas entre os ambientes estudados (Tabela 4), o que demonstra que, apesar de alterações na composição e densidade, a 
biomassa da floresta tende a se manter, provavelmente desencadeada pelas taxas de crescimento, que sofreram influência positiva devido a abertura de clareiras.

Bezerra et al. (2018) e Vatraz et al. (2015) observaram esse mesmo comportamento em área de manejo florestal. Os autores verificaram que os indivíduos arbóreos expostos à luz apresentaram maior crescimento em diâmetro do que as árvores parcialmente e totalmente sombreadas.

Tabela 4: Ganhos e perdas de estoques em Unidades de Produção Anual (UPA) submetidas a diferentes taxas de corte na Unidade de Manejo Florestal III, Flona do Jamari, RO.

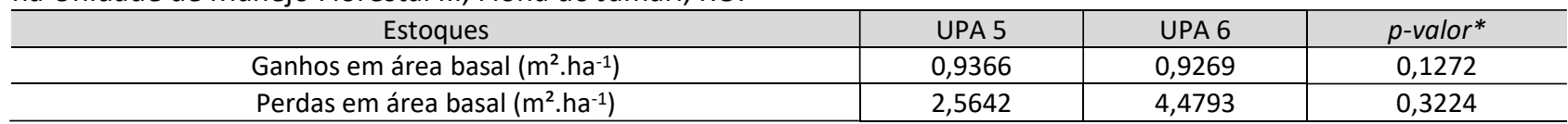

Onde: *teste t a $95 \%$ de probabilidade.

A maior perda de estoque é esperada nos primeiros anos após exploração, pois a colheita das árvores de maior porte e taxas de mortalidade contribuem para esse cenário. Destaca-se ainda, que as entradas de estoque proveniente das árvores ingressantes são baixas, devido aos baixos diâmetros desses indivíduos, refletindo em pouca influência no ganho de estoque em biomassa.

No entanto, o monitoramento a médio e longo prazo será importante para entender em que momento o ganho em biomassa será maior que a perda, pois conforme Puig (2008), ao longo do tempo, a taxa de mortalidade tende a reduzir por causa exploratória e, o ingresso passa a aumentar, assim como o crescimento favorecido pela abertura de clareiras, contribuindo para o ganho de estoque da floresta.

\section{CONCLUSÕES}

A retirada de madeira na Unidade de Produção Anual (UPA) 5 causou pouca alteração na composição e estrutura da floresta após exploração. Na UPA 6 a incidência de parcelas mais próximas as áreas de colheita, favoreceu para a ocorrência de danos, causando maior mortalidade de árvores. As espécies raras se mostraram sensíveis as taxas de corte, sendo necessário nos planos de manejo, maior atenção a esses grupos, que são importantes para biodiversidade dos ecossistemas amazônicos. A taxa de mortalidade foi maior que o ingresso em ambas as Unidades de Produção Anual, sendo os indivíduos do sub-bosque os mais afetados, que favoreceu juntamente com as taxas de colheita, maior perda de estoque após exploração.

AGRADECIMENTOS: Instituto Chico Mendes de conservação da Biodiversidade (ICMBio), e a Empresa Amata.

\section{REFERÊNCIAS}

ALVARES, C. A.; STAPE, J. L.; SENTELHAS, P. C.; MORAES, G.; LEONARDO, J.; SPAROVEK, G.. Köppen's climate classification map for Brazil. Meteorologische Zeitschrift, v.22, n.6, p.711728, 2013.

AMATA. Plano de Manejo Florestal Sustentável. AMATA, 2009.

BEZERRA, T. G.; LIMA, A. O. S.; ARAÚJO, J. T. R.; SANTOS, M. G. S.; NEVES, R. L. P.; MORAES, G. C.; MELO, L. O.. Estrutura e dinâmica de uma área manejada na Floresta Nacional do
Tapajós. Revista Agroecossistemas, v.10, n.2, p.94-112, 2018.

BRASIL. Ministério do Meio Ambiente. Plano de Manejo da Floresta Nacional do Jamari. Brasília: MMA, 2005.

BRASIL. Ministério do Meio Ambiente. Resolução Conama $\mathbf{n}$. 406, de 02 de fevereiro de 2009. Estabelece parâmetros técnicos a serem adotados na elaboração, apresentação, avaliação técnica e execução de Plano de Manejo Florestal Sustentável-PMFS com fins madeireiros, para florestas 
nativas e suas formas de sucessão no bioma Amazônia Brasília: MMA, 2009

CASTRO, M. S. A.; VIANA, A. P. S.; LOBATO, C. C.; MUNIZ, J. L.; LIMA, M. W. S.; MELO, L. O.. Impactos da exploração florestal na estrutura e na composição florística da vegetação remanescente em área manejada na Floresta Nacional do Tapajós, Belterra, Pará. Revista

Agroecossistemas, v.10, n.2, p.125-135, 2018.

D'OLIVEIRA, M. V. N.; OLIVEIRA, L. C.; ACUÑA, M. H. A.; BRAZ, E. M.. Twenty years monitoring growth dynamics of a logged tropical forest in Western Amazon. Pesquisa Florestal Brasileira, v.37, n.92, p.493-502, 2017.

OLIVEIRA, K. B.; REZENDE, A. V.; FREITAS, L. J. M.; MURTA JÚNIOR, L. S.; BARROS, Q. S.; COSTA, L. S.. Monitoramento da estrutura e caracterização ecológica em floresta tropical manejada na Amazônia Brasileira. Ciências Florestais, v.14, n.4, 2019

DIONÍSIO, L. F. S.; SCHWARTZ, G.; LOPES, J. C.; OLIVEIRA, F. A.. Growth, mortality, and recruitment of tree species in an Amazonian rainforest over 13 years of reduced impact logging. Forest Ecology and Management, v.430, p.150-156, 2018.

HIRAI, E. H.; CARVALHO, C. J. R.; SILVA, J. N. M.; CARVALHO, J. O. P.; QUEIROZ, W. T.. Efeito da exploração florestal de impacto reduzido sobre a regeneração natural em uma floresta densa de terra firme no município de Paragominas na Amazônia brasileira. Scientia Forestalis, Piracicaba, v.40, n.95, p.306-315, 2012.

IVANAUSKAS, N. M.; MONTEIRO, R.; RODRIGUES, R. R.. Estrutura de um trecho de floresta Amazônica na bacia do alto rio Xingu. Acta Amazônica, v.34, n.2, p.275-299, 2004.

KROESSIN, A.. Estrutura e dinâmica de uma floresta tropical submetida à exploração de impacto reduzindo em Belterra, Pará. Dissertação Mestrado. Manaus: Universidade Federal do Amazonas, 2013.

LIMA, B. A.; ALMEIDA, B. R. S.; SOUZA, E. A. B.; CRUZ, G. S.; MELO, M. B.; MELO, L. O.; COSTA, D. L.; SANOS, M. F..
Estrutura e dinâmica florestal sob efeito do manejo madeireiro na FLONA Tapajós. Advances in Forestry Science, v.5, n.4, p.437-443, 2018.

PUIG, H.. A Floresta Tropical Úmida. São Paulo: UNESP, 2008

R CORE TEAM. R: A language and environment for statisticalcomputing. Vienna: R Foundation for Statistical Computing, 2019.

RONDÔNIA. Secretaria de Estado do Desenvolvimento Ambiental. Boletim Climatológico de Rondônia, ano 2008. Porto Velho: SEDAM, 2010

RUY, C. C.; WEISER, N. H.; PIAZZA, G. E.; CORREIA, J.; ROBERT, R. C. G.. Danos decorrentes do uso da floresta baseado em conhecimentos tradicionais: um estudo de caso em uma floresta secundária de Santa Catarina. Enciclopédia Biosfera, Goiânia, v.10, n.19, p.2869, 2014.

SBF. Serviço Florestal Brasileiro. Balanço de produção: Jamari (RO). SBF, 2019.

SILVA, J. N. M.; LOPES, J. C. A.; OLIVEIRA, L. C.; SILVA, S. M. A.; CARVALHO, J. O. P.; COSTA, D. H. M.; MELO, M. S.; TAVARES, M. J. M.. Diretrizes para instalação e medição de parcelas permanentes em florestas naturais da Amazônia Brasileira. Belém: Embrapa Amazônia Oriental, 2005.

SOUZA, A. L.; SOARES, C. P. B.. Florestas Nativas: estrutura, dinâmica e manejo. Viçosa: UFV, 2013.

VATRAZ, S.; CARVALHO, J. O. P.; SILVA, J. N. M.; CASTRO, T. C.. Efeito da exploração de impacto reduzido na dinâmica do crescimento de uma floresta natural. Scientia Forestalis, Piracicaba, v.44, n.109, p.261-271, 2016.

VELOSO, H. P.; RANGEL FILHO, A. L. R.; LIMA, J. C. A.. Classificação da vegetação brasileira, adaptada a um sistema universal. Ministério da Economia, Fazenda e Planejamento, Fundação Instituto Brasileiro de Geografia e Estatística, Diretoria de Geociências, Departamento de Recursos Naturais e Estudos Ambientais, 1991.

A CBPC - Companhia Brasileira de Produção Científica (CNPJ: 11.221.422/0001-03) detém os direitos materiais desta publicação. Os direitos referem-se à publicação do trabalho em qualquer parte do mundo, incluindo os direitos às renovações, expansões e disseminações da contribuição, bem como outros direitos subsidiários. Todos os trabalhos publicados eletronicamente poderão posteriormente ser publicados em coletâneas impressas sob coordenação da Sustenere Publishing, da Companhia Brasileira de Produção Científica e seus parceiros autorizados. Os (as) autores (as) preservam os direitos autorais, mas não têm permissão para a publicação da contribuição em outro meio, impresso ou digital, em português ou em tradução. 